\title{
An Online Review Research Based on Opinion Dynamics Model
}

\author{
Zizhan Lin ${ }^{1}$, Jinlian Zhou ${ }^{2}$, Ye Wu ${ }^{2}$, Jinghua Xiao ${ }^{1}$ \\ ${ }^{1}$ School of Science, Beijing University of Posts and Telecommunications, Beijing 100876 \\ ${ }^{2}$ School of Journalism and Communication, Beijing Normal University, Beijing 100875
}

Abstract: Due to the rapid growth of Internet, consumers obtain information about products and experiences from online rating platforms, which further influences their purchase decisions. Considering the differences in the opinion interaction pattern, traditional opinion dynamics models cannot explain individual review behaviors. In order to explore the evolution process of opinions on the online review platform, we optimized the network structure and confidence thresholds of traditional models based on the collected data from Dianping, and created an opinion dynamics model for online rating platforms. The proposed method can advance the understanding for the evolution process of online opinion.

Keywords: Complex Network; Opinion Dynamics; Hegselmann-Krause Model; Online Review; Opinion Evolution

\section{Introduction}

In the network environment, individuals are not only consumers of social information, but also producers of information and dissemination of ideas. Online reviews, as a way of expressing individual opinions, refer to users or consumers directly dealing with business through online platforms.

Due to the rise of social media, the evolution mechanism of public opinion on the Internet has become the focus of opinion dynamics research. At present, the research on the dynamics of individual online comments mainly uses the method of multi-agent modeling to explain the distribution of comments or forecast. The research by Qu Mingyang and Huang Feihu believes that in the process of film review scoring, individual opinions will be influenced by film quality, other uses. The influence of household rating and their own rating habits, thus establishing a movie rating influence model, which can better predict the movie opinion distribution of film score ${ }^{[1]}$ different from the prediction of scoring opinions, Su Jiong-ming and others established the motivation of continuous opinions. Learn the model, judge whether the individual joins the scoring group or not, and perform scoring behaviors, thus predicting the number of online reviews ${ }^{[2]}$. The study by Wan Yan et al. takes into account the limitations of individual reading attention, the possibility of publishing comments and the limited trust threshold. On the basis of response factors, this paper puts forward a dynamic model of online comment evolution suitable for e-commerce environment.

The existing viewpoint dynamic models can be roughly divided into two types: discrete model and continuous viewpoint model. Among them, the discrete model's viewpoint is described as a binary value of "support" or "objection". The representative models include Sznajd model ${ }^{[4]}$, Majority rule model ${ }^{[5]}$, Voter model ${ }^{[6]}$, etc. Among the continuous models, the representative ones are Deffuant models based on bounded trust ${ }^{[7]}$ and Hegselmann-Krause Mode ${ }^{[8]}$. At present, the research on the dynamics of public opinion is mainly based on the traditional Deffuant model and HK model, and it is studied from the perspectives of social network structure ${ }^{[9-17]}$.

With the rise of social media, the evolution mechanism of public opinion in social networks has become the focus of viewpoint dynamics research. However, due to the differences in the dissemination modes of basic opinions, the

This is an open-access article distributed under the terms of the Creative Commons Attribution Non-Commercial License (http://creativecommons. org/licenses/by-nc/4.0/), which permits unrestricted non-commercial use, distribution, and reproduction in any medium, provided the original work is properly cited. 
viewpoint dynamics model of the evolution of network public opinion is not fully applicable to individuals. Research on Line Comment Behavior. In contrast, the dissemination of opinion in the review mode has the following characteristics: (1) Research on the evolution of public opinion. After the exchange of opinions between Chinese individuals, the opinions of the exchanged individuals may change. The dissemination of views is not interactive, only the views of the first commentator will have an impact on the views of the second commentator; (2) Most of the research is based on the simulation of real social networks, usually taking into account network structural features or special sections; (3) Social networks can communicate with friends and strangers as well. (4) The evolution of public opinion, the exchange of individual opinions in the review mode will also be affected.

\section{Public comments}

Public comment is one of the largest online comment platforms in China. Users can contribute their comments on merchants on the platform.At the same time, they can also browse other users' comments to help them make their own consumption decisions. The number of transactions exceeded 58 billion, the transaction amount 357 billion yuan, providing catering, entertainment, shopping and other services for the country 2800 cities and counties 310100 million annual transaction user contracts 4.4 million annual active merchants.

We have selected shops with more than 500 reviews, totaling 1610, homes, 2056387 and reviews. The rating data of users in public reviews include: star rating, taste, environment and service, all of which are scores of 10, 20, 30, 40, 50 and the average of these four ratings is taken as the real rating. Here we do not consider the extreme score of 50 points, because: (1)In order to improve public praise and attract more users to spend, merchants usually employ water troops to fake high-score comments as ordinary customers ${ }^{[18]}$; (2) Some users will choose 50 points by default when commenting; These two situations do not reflect the real views of users.

By observing the distribution of store ratings for these 1610 ,more than 500 and 500 reviews, we found that the distribution of store ratings for public reviews mainly includes two modes: single opinion group and two opposing opinion groups. We selected six stores from the two models and plotted their score distribution, as shown in Figure 1.
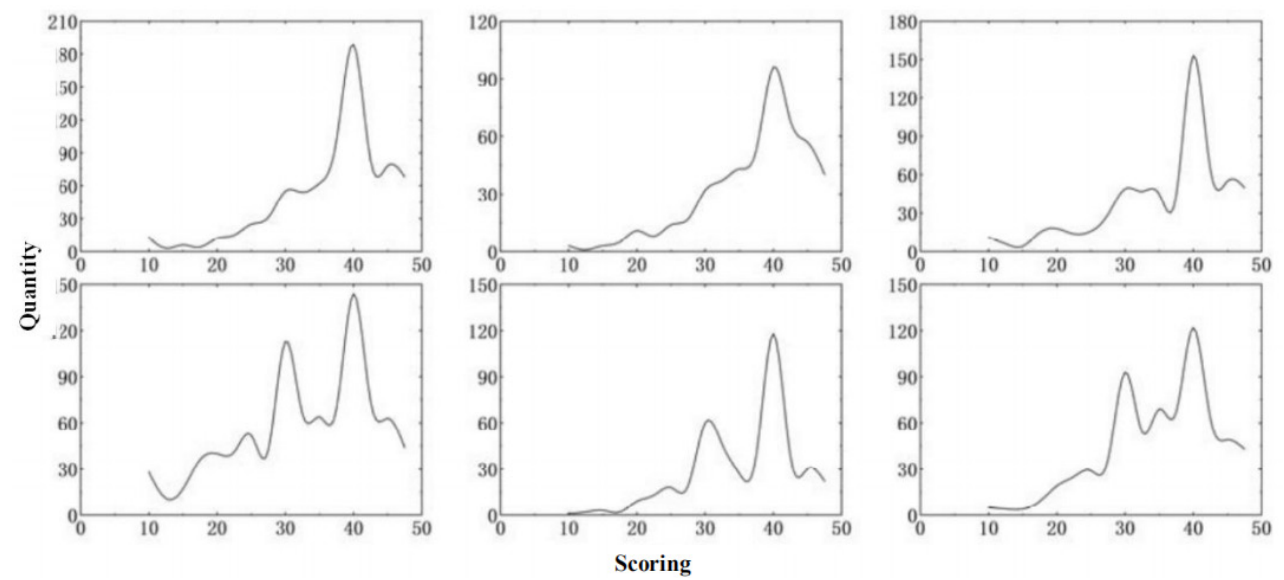

Figure 1. The scoring distribution of selected stores.

\section{Model building}

\subsection{Hegselmann-Krause model}

Traditional HK model is a continuous dynamic model based on bounded trust. Its purpose is to study the expression of individual opinions in a group. Assuming that a group contains $n$ individuals, representing the individual's point of view value at the moment, the individual will interact with other individuals in the group. If the difference between the two individuals is less than the point of view threshold, that is, the individual will have an impact on the individual, otherwise there will be no impact.

\subsection{Model revision}

(1) The viewpoint interaction between individuals in the model is based on a complex network, and only neighboring nodes are possible. Exchange of views. However, in online reviews, social networks are not closely related, 
and the exchange of views among individuals is not two-way.It is based on the chronological order of the reviews. Only the opinions of the first reviewer will affect the opinions of the second reviewer. Therefore, we changed the network structure to a one-way network structure in chronological order.

(2) Online merchants may have a large number of reviews, and users usually do not browse due to the limited time and energy of individuals. All the comment data, therefore, only some recent comments will have an impact in the process of view exchange.

(3) The same individual does not have the same degree of trust in the opinion values of other individuals in the group. The trust weight of individual opinion value is randomly generated. In public comments, comments that users have not seen do not affect users. At the same time, the timeliness of comments leads to different trust levels of users to different comments, and recent comments are a reference to users. The significance is much greater than the relatively early comments.

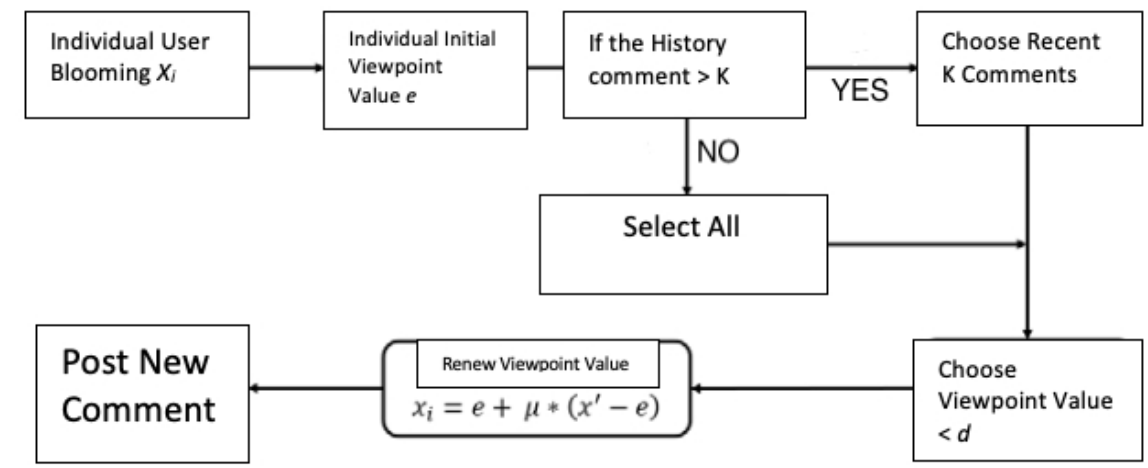

Figure 2. The flowchart of the model.

\subsection{Model simulation}

Based on the HK model, we propose a new model suitable for online review mode according to the actual situation of online review, and explore the influence of various parameters in the new model on the evolution of views through simulation.

(1) As shown in Figure 3, when the value of parameter $\mathrm{k}$ is small, the number of views that the user chooses to refer to when scoring the store is small. Therefore, other people's ratings have limited influence on the users views. As the values of $\mathrm{K}$ and $\mathrm{K}$ increase, the number of user-selected reference scores increases, which is more easily influenced by the opinions that the publishing time is closer to the current time, thus reaching agreement with the opinions of the surrounding users and forming an opinion group; When the values of $\mathrm{K}$ and $\mathrm{K}$ are greater than 10 , the changes of the values of $\mathrm{K}$ and $\mathrm{K}$ " will not lead to obvious differences in results.
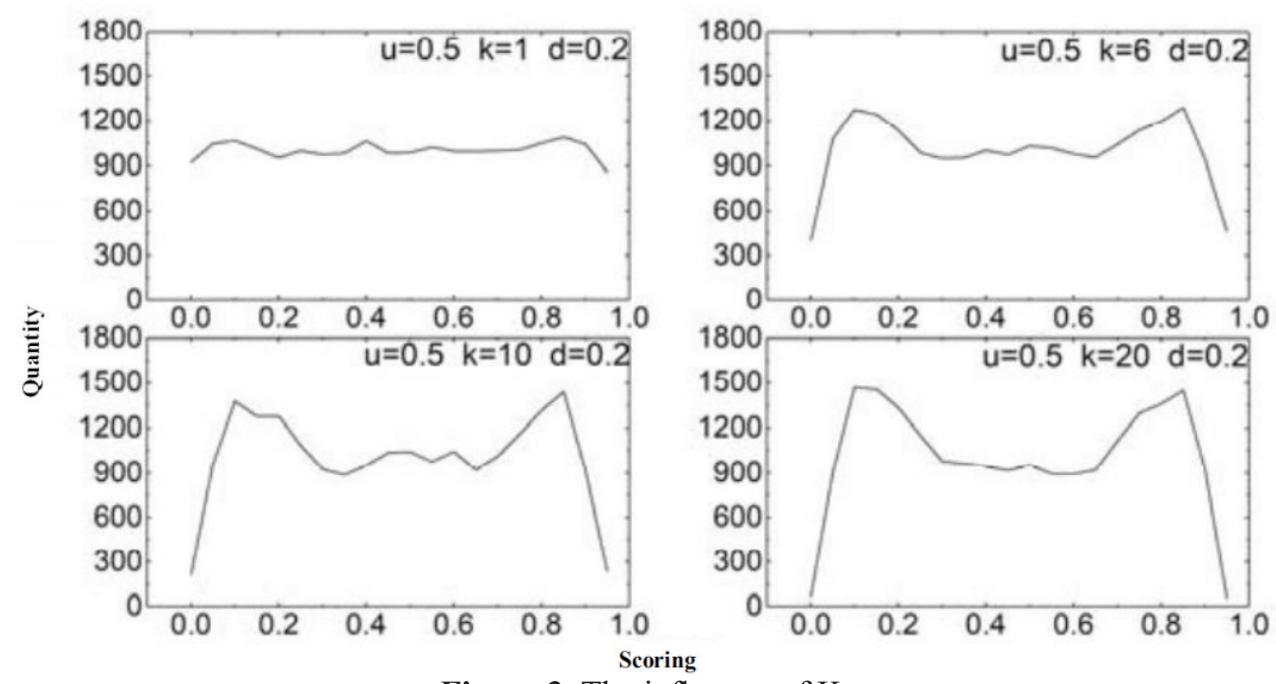

Figure 3. The influence of $\mathrm{K}$. 
(2) Opinion difference threshold $(\mathrm{d})$ is the main factor affecting the final result. As shown in Figure 4, when $d=0$, it is obvious that there is no mutual influence between individual opinions, and the results satisfy the uniform distribution. With the increase of threshold value and D, the opinions of individuals are mutual. Impact, there will be some individual views reached an agreement, eventually forming different opinion groups.

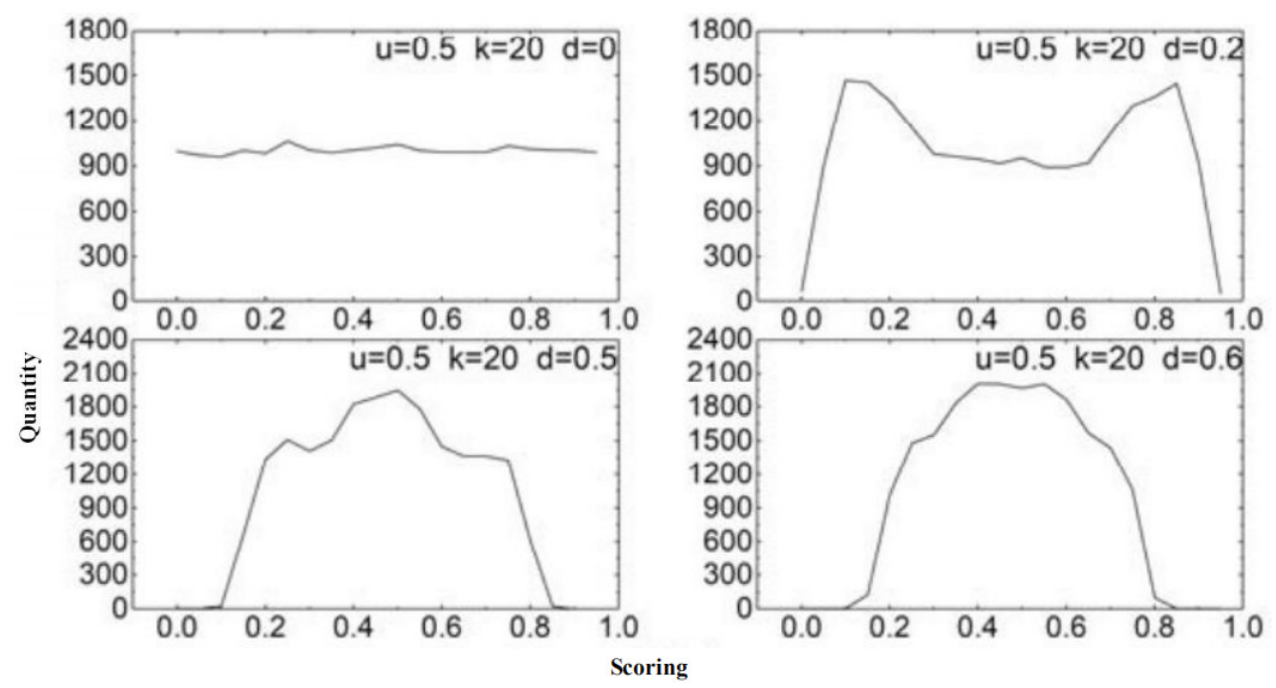

Figure 4. The influence of $d$.

(3) Convergence parameter $\mu$ indicates the degree of trust that individuals have in other individuals' viewpoints. As shown in Figure 5, when the mu value is small when the individual is not easy to be affected by others, as mu value increases, the individual is affected by others.

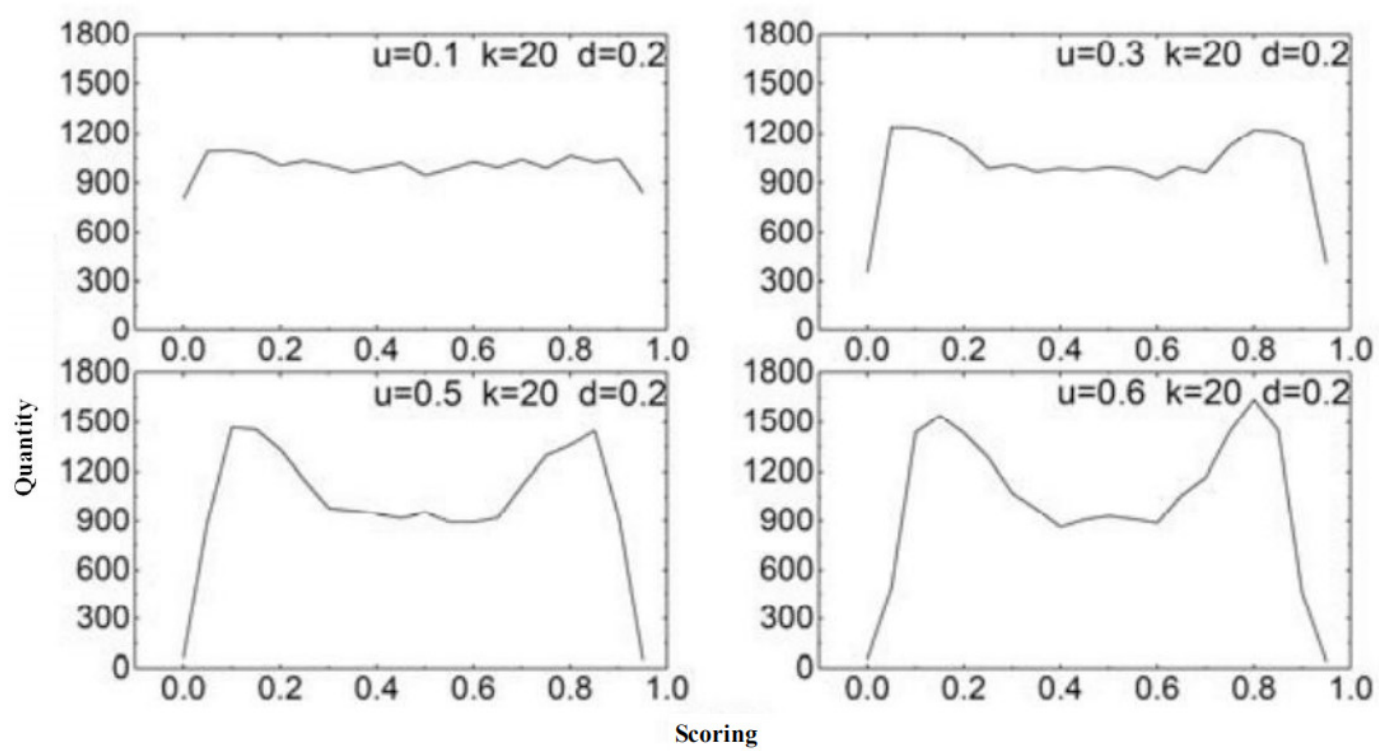

Figure 5. The influence of $\mu$ false.

From the above simulation results, we can see that the evolution of opinions in online reviews depends on the number of individual reference reviews $\mathrm{k}$ and viewpoint threshold, $\mathrm{D}$, and convergence parameter $\mu$. Among them, opinion threshold value $\mathrm{D}$ are the main factors that affect the number of final opinion groups. The two evolution results in the simulation: a single mainstream opinion and two kinds of opinion groups are consistent with the actual store score distribution we observed in public comments.

\section{Conclusion}

The emergence of online comment platforms such as public comment and Taobao has enabled users to share their views and ideas with others. It is also easy to be influenced by others. The traditional public opinion dynamics model explores the evolution of viewpoints through social interpersonal networks. The internal mechanism of. However, the 
network of interpersonal relationships on the online review platform is very thin, users do not know each other, and traditional geography.

(1) Replace the interpersonal network with a one-way transmission structure in chronological order; (2) The simulation results show that in the online review mode, the results of opinion evolution are influenced by the number of reviews produced.

\section{References}

1. By Mingyang ,Huang Feihu; Movie Online Review Forecast Based on Viewpoint Dynamics[J]; Modern Computer; : China Xunkan, 2016 (2): 7-11.

2. Su Jiong-ming, Liu Baohong, Li Qi, et al. Prediction of Number of Online Comments Based on Viewpoint Dynamics [J]. Computer Engineering, 2014, 40(10): 155-160.

3. WAN Y, MA B, PAN Y. Opinion evolution of online consumer reviews in the e-commerce environment[J]. Electronic Commerce Research, 2018, 18(2): 291-311.

4. SZNAJD-WERON K, SZNAJD J. Opinion evolution in closed community[J]. International Journal of Modern Physics C, 2000, 11(06): 1157-1165.

5. HOWE D A, PERCIVAL D B. Wavelet variance, Allan variance, and leakage[J]. IEEE transactions on instrumentation and measurement, 1995, 44(2): 94-97.

6. HOLLEY R A, LIGGETT T M. Ergodic theorems for weakly interacting infinite systems and the voter model[J]. The annals of probability, 1975: 643-663.

7. DEFFUANT G, NEAU D, AMBLARD F, et al. Mixing beliefs among interacting agents[J]. Advances in Complex Systems, 2000, 3(01n04): 87-98.

8. HEGSELMANN R, KRAUSE U. Opinion dynamics and bounded confidence models, analysis, and simulation[J]. Journal of artificial societies and social simulation, 2002, 5(3): 1-24.

9. PROSKURNIKOV A V, TEMPO R. A tutorial on modeling and analysis of dynamic social networks. Part II[J]. Annual Reviews in Control, 2018, 45: 166-190.

10. MENG X F, VAN GORDER R A, PORTER M A. Opinion formation and distribution in a bounded-confidence model on various networks[J]. Physical Review E, 2018, 97(2): 022312.

11. LI D, HAN D, MA J, et al. Opinion dynamics in activity-driven networks[J]. EPL (Europhysics Letters), 2018, 120(2): 28002.

12. PEREZ I J, CABRERIZO F J, ALONSO S, et al. On dynamic consensus processes in group decision making problems[J]. Information Sciences, 2018, 459: 20-35.

13. Gou Zhijian, Fan Mingyu, Wang Guangwei, Research on Evolution of Continuous Views Without Trust Boundary Limits in Complex Networks, [J] Journal of University of Electronic Science and Technology, 2015, 44(5): 749-756.

14. Kan Jiaqian, Xie Jiarong, Zhang Haifeng ;Analysis of the Influence of Social Strengthening Effect and Linking Weight on Network Information Dissemination ; $[\mathrm{J}]$ University of Electronic Science and Technology Newspaper, 2014, 43(1): 21-25.

15. LEE J, PARK D H, HAN I. The effect of negative online consumer reviews on product attitude: An information processing view[J]. Electronic commerce research and applications, 2008, 7(3): 341-352.

16. GHOSE A, IPEIROTIS P G. Estimating the helpfulness and economic impact of product reviews: Mining text and reviewer characteristics[J]. IEEE Transactions on Knowledge and Data Engineering, 2011, 23(10): 1498-1512.

17. Sean, Yang Shan, Li Xia. Defuant-I Public Opinion Communication Model with Individual Information Difference [J]. Systems Engineering, 2017(6):82-88.

18. JINDAL N, LIU B. Opinion spam and analysis[C]//Proceedings of the 2008 international conference on web search and data mining. ACM, 2008: 219-230. 